\title{
Surgical treatment of Zenker's diverticulum. Is the open approach really outdated?
}

Authors:

Szabó K., Szentkereszty Zs.

University of Debrecen, Clinical

Center, Institute of Surgery,

Debrecen, Hungary

Address: Moricz Zsigmond Krt.

22, 4032 Debrecen, Hungary

E-mail:szakaga@yahoo.com

Keywords: Zenker diverticulum, Transcervical open surgery

\begin{abstract}
The authors aimed to elucidate the indications of transcervical surgical approach and also to review the literature in the treatment of symptomatic Zenker's diverticulum (ZD), focusing on clear indications for open surgery (diverticulectomy with cricopharyngeal myotomy). The authors analyzed the efficacy of open surgery versus the current standard endoscopic diverticulotomy, focusing on the size of the diverticulum, surgical approach, and postoperative morbidity.
\end{abstract}

Following a retrospective search in the literature using Medline and PuBMed database of the past 5 years, the authors found 79 articles about the treatment of symptomatic Zenker's diverticulum (keywords: Zenker's diverticulum, open surgery, endoscopic diverticulectomy). 25 papers were included; the exclusion criteria were small number of patients, non-English publications.

Although endoscopic diverticulectomy is the preferred method of treatment in symptomatic Zenker's diverticulum, in those cases, in which the patient is not eligible for endoscopic treatment, open surgery is a safe and reliable choice when treating a small ZD. 


\section{Introduction}

Zenker's diverticulum, or cricopharyngeal diverticulum, is an outpoutching of the pharyngeal mucosa in the dorsal wall, located at the junction of the hypopharynx and the esophagus. The anatomical site is also known as Killian's triangle, the borders are formed superiorly by the fibers of the lower inferior pharyngeal constrictor and the transverse fibers of the cricopharyngeus muscle. (Aiolfi et.al 2015, Aquino et al 2015, Babur et al 2014, Barton et al 2016, Bizotto et al 2013, Bobkiewicz et al 2015, Courson et al 2013, de la Morena et al 2016,Herrero et al 2012, Jackson et al 2016 Figure 1.)

The diagnosis is based on typical complaints, such as dysphagia, regurgitation, coughing from aspiration, chronic aspiration pneumonia and barium swallow esophagogram. For classification of cycopharingeal diverticulums the Bombard classification is used. The cornerstone of modern treatment in sympomatic Zenker's diverticulum is the crycopharingeal myotomy.

Although in the last three decades the basic treatment of these diverticulums is endoscopic surgery we found only a few publications about the so called conventional surgery, which offers the lowest rate of recurrence.

\subsection{Surgical technique}

The operation is usually performed under general anesthesia, although other methods can also be performed. The patient is in a supin position, the head hyperextended and turned to the right side. The left lateral neck incision runs ventrally to the sternocleidomastoid muscle. After dividing the platysma, the pharynx and cervical esophagus are exposed by retracting the sternocleidomastoid and carotid sheat laterally the larynx and thyroid gland medially. After identification of the pouch, a complete dissection from the surrounding tissue is performed until the neck of the pouch is displayed. Myotomy 
of crycopharyngeal and esophageal muscle is performed for a length of approximately $5 \mathrm{~cm}$ on the cervical esophagus. Following myotomy, resection of the diverticulum is usually performed with a stapler. (Mantsopoulos et al 2012 Figure 2.)

During the operation, great care must be given in order to avoid injury to the following structures: recurrent laryngeal nerve, external laryngeal nerve, descending hypoglossal nerve, cervical cutanous nerve. A drain is placed, the platysma and subcutaneous tissue are sutured and the skin incision closed. (Mantsopoulos et al 2012)

\section{Methods:}

A retrospective review of Medline and PubMed databases of the past 5 years using the search terms ,Zenker's diverticulum, pharyngeal pouch, open surgery, endoscopic diverticulectomy". A total of 25 relevant publications were found. We focused mainly on publications discussing open surgery.

\section{Included papers}

The search provided 25 relevant articles. Almost all papers compared different endoscopic treatment with transcervical approach, focusing on postoperative complications, with only minimal discussion of transcervical resection. We selected publications which discussed in detail the positive effects of open surgery

\section{Indications for open/}

\section{transcervical surgery}

The basis of both endoscopic and conventional, transcervical surgical approach is the crycopharingeal myotomy. (Verdonk et al, 2015, Mantsopoulos et al 2012, de la Morena et al 2016, Barton et al 2016, Jones et al 2016, Seth et al 2014, Shahawy et al 2014,) In the last decades several modified techniques of endoscopic oesophago-diverticulostmy were developed. Although nowadays the endoscopic treatment is the first of choice when treating Zenker's diverticulum, there are some indications for open 
transcervical operations. Other indications for open surgery include: technical failure of endoscopic surgery, difficulty in placing the diverticuloscope, usually a consequence of neck rigidity, short neck, dental anomalia, lateral position of the diverticulum, dysphagia, squamous cell carcinoma. Perforation which is a rare complication, usually needs urgent intervention. However, in selected patients, with no signs of mediastinitis, sepsis, pleural effusion, good general status, conservative treatment can be successful. (Herrero et al 2013).

\subsection{Physical habitus}

When the patient is eligible, endoscopic treatment is usually surgeons' first choice. However, knowing the fact that Zenker's diverticulum is a disease of the elderly, mean age category is about 70, the placement of diverticuloscope can be difficult because of body habitus, neck rigidity and vertebral disease. In these cases, open surgery should be considered. (Verdonk et al 2015, Mantsopoulos et al 2012, de la Morena et al 2016, Barton et al 2016, Kannabiran et al 2015, Maca et al 2013, Yuan et al 2013, Visser et al 2016)

\subsection{Size of diverticulum}

One of the most accepted indication for open surgery is the large size of diverticulum. The authors agree that open surgery is indicated if the size of the diverticulum is bigger than $6 \mathrm{~cm}$. To classify the Zenker's diverticulum, the Bombard and Morton \& Bartley Classification was developed. The Bombard III-IV. and Morton \& Bartley III. cases are candidates for open surgery. In these cases the endoscopic surgery is not always successful.

\subsection{Technical failure of}

\section{endoscopic surgery}

Following endoscopic laser diverticulotomy (ELD) and endoscopic stapler diverticulotomy (EST) the most common complication was subcutaneous emphysema, dental injury, throat pain, incomplete perforation of the esophagus. One patient required immediate reoperation because of bleeding. (Jiang et 
al 2016) The complications related to the transcervical approach are rare. The most common complication is the fistula formation. It seems to be significantly higher $(4 \%)$ after open surgery than $(1 \%)$ after endoscopic operations. (Verdonk et al 2015)

\subsection{Other rare indications}

The injury of the big neck vessels in these cases during endoscopy is high so cervical exploration of the diverticulum is needed.

In young patients who present with no or few comorbidities and knowing that reoccurrence of symptoms is higher after endoscopic diverticulostomy, transcervical diverticulectomy is the preferred approach for the treatment of Zenker's diverticulum. (Nuno Guzman et al 2014)

\section{Complications of open}

\section{transcervical surgery}

The complications related to the transcervical approach are rare. The most common complication is the fistula formation. It seems to be significantly higher (4\%) after open surgery than (1\%) after endoscopic operations. (Verdonk et al 2015, Mantsopoulos et al 2012, de la Morena et al 2016, Barton et al 2016, Seth et al 2014, Bizotto et al 2013) The method used to resect the $\mathrm{ZD}$ could also play a role, as using mechanical suture seems to offer advantages, compared with the manual one, for having demonstrated lower rates of dehiscence and stenosis of the pharyngeal suture. (Aquino et al 2015)

Recurrent nerve palsy and haematoma in the operation site are more common after open surgery. (Verdonk et al 2015, Mantsopoulos et al 2012, Barton et al 2016,Leibowitz et al 2014)

In contrast of the above mentioned complications, the rate of emphysema and mediastinitis are lower after transcervical surgery. (Verdonk et al 2015)

\section{Length of hospital stay (LOS)}

\section{after open transcervical surgery}

The LOS after open surgery is higher than after endoscopic surgery (Mantsopoulos et al 2012, de la Morena et al 2016, Barton et al 2016, Seth et al 2014, 
Bizotto et al 2013, Yuan et al 2013, Jiang et al 2016, Maca et al 2013, Sonbare et al 2015). In a systematic review of Verdonk et al., the LOS was significantly higher after open than endoscopic surgery. (Verdonk et al 2015)

\section{Results of open transcervical}

\section{surgery}

When comparing the primary outcome, the failure rate is significantly lower in open surgery than after endoscopic techniques. These failures are secondary to difficult exposure of ZD. (Bizotto et al 2013, Jackson et al 2016, Seth et al 2014, Verdonk et al 2015,)

The high effectiveness of the surgical method, related to the low risk of recurrence, demands that open surgery figures among the effective methods of treatment. Despite the higher risk of perioperative complications, it seems that general surgical risk in clinical centers with high experience in treating $\mathrm{ZD}$ is comparable to the risk undertaken when using endoscopy as a treatment modality. (Bobkiewicz et al 2015) When comparing post-operative complications that could be caused by extended anesthesia, such as urinary retention, GI problems, cardiac issues, pulmonary atelectasis, these occur at a similar rate after endoscopic treatment. (Yuan et al 2013)

\section{Conclusion}

Although endoscopic diverticulectomy is the preferred method of treatment in symptomatic Zenker's diverticulum, in those cases, in which the patient is not eligible for endoscopic treatment, open surgery is a safe and reliable choice when treating a small ZD. 


\section{References}

- Aiolfi A, Scolari F, Saino G, et al.: Current status of minimally invasive endoscopic management for Zenker diverticulum. World J Gastrointest Endosc. 2015; 7(2):87-93. doi: 10.4253/wjge.v7.i2.87.

- Aquino J, Chagas J, Said M, et al.: Assessment of mechanical and manual suture in thes surgical treatment of the pharyngoesophageal diverticulum. ABCD Arq Bras Cir Dig 2015; 28(4):239-242.

- Babur T.: Iatrogenic Zenker's diverticulum perforation: A conservatively treated case. Ulusal Cer Derg 2014; 30:234-236.

- Barton MD, Detwiller KY, Palmer $\mathrm{AD}$, et al.: The safety and efficacy of endoscopic Zenker's diverticulotomy: A cohort study. Laryngoscope, 2016; doi:10.1002/lary.26046.

- Bizotto A, Lacopini F, Landi R, et al.: Zenker's diverticulum:exploring treatment options. Acta Otorhinolaryngol Ital. 2013; 33(4):219-29.

- Bobkiewicz A, Banasiewicz T, Krokowicz L, et al.: Assessment of the results of surgical treatment of
Zenker's diverticulum in own material. Polski Przeglad Chirurgiczny 2015; 87(3):109-115.

- Courson AM, Landry AM, Lott DG, et al.: Open Staple diverticulectomy for a large recurrent Zenker's diverticulum: still a valid procedure. Ann Otol Rhinol Laryngol. 2013; 122(12):729-33.

- de la Morena Madrigal EJ, Pérez Arellano E, Rodríguez García I.: Flexible endoscopic treatment of Zenker's diverticulum: thirteen years'experience in Spain. Rev Esp Engerm Dig. 2016; 108(6):297-303. doi:10.17235/reed.2016.4030/2015

- Herrero EA, Pérez DL, Tejero-Garcés GG, et al.: Treatment of Zenker's diverticulum: comparison of different techniques. Acta Otorrinolaringol Esp. 2013; 64(1):1-5. doi: 10.1016/j.otorri.2012.06.002.

- Jackson B, Ahmad Z, Morton RP.: Utility of transcervical management of Zenker's diverticulum. J Laryngol Otol. 2016; 130 Suppl 1:S16-9. doi: 0.1017/S0022215115003205

- Jiang N.. Sung CK, Damrose EJ.: Improvement in the Reflux Symptom 
Index Following Surgery for Cricopharyngeal Dysfunction. J Voice. 2016; pii: S08921997(16)00040-0. doi: 10.2016/j.voice.2016.02.006.

- Johanson CM, Postma GN.: Zenker Diverticulum-Which surgical Approach is Superior? JAMA Otolaryngol Head Neck Surg. 2016; 142(4):401-3. doi: $0.1001 /$ jamaoto.2015.3892

- Jones D, Aloraini A, Gowing S., et al.: Evolving Management of Zenker's Diverticulum int he Endoscopic Era: A North American Experience. World J Surg. 2016; 40(6):1390-6. doi: 10.1007/s002680163442-0

- Kannabiran V, Gooey J, Fisichella P.: A tailored approach to the Surgical Treatment of Zenker's Diverticula. J Gastroinetst Surg 2015; 19:949-954.

- Kannabiran VR, Gooey J, Fisichelle PM.: A Tailored Approach to the Surgical Treatment of Zenker's Diverticula. J Gastrointest Surg. 2015; 19(5):949-54. doi: 10.1007/s11605-015-2774-7.

- Leibowitz JM, Fundakowski CE, Abouyared M, et al.: Surgical Tecniques for Zenker's Diverticulum:Comparative Analysis
Otolaryngol Head Neck Surg. 2014, 151(1):52-8.

doi:

10.1177/0194599814529405.

- Mantsopoulos K, Psychogios G, Künzel $\mathrm{jJ}$ et al.: Evaluation of the diferent transcervical approaches for Zenker's diverticulum. Otolaryngol Head Neck Surg 2012; 146:725-9.

- Nuno-Guzmán CM, García -Carrasco $\mathrm{D}$, Haro $\mathrm{M}$, et al.: Zenker's Diverticulum: Diagnostic Approach and Surgical Management. Case Rep Gastroenterol. 2014; 8(3):206-12. doi:10.1159/000369130.

- Seth R, Rajasekaran K, Lee WT, et al.: Patient reported outcomes in endoscopic and open transcervical treatment for Zenker's diverticulum. J Laryngoscope. 2014; 124(1):119-25. doi: 10.1002/lary.24152.

- Shah RN, Slaughter KA, Fedore LW, et al.: Does residual wall size or technique matter in treatment of Zenker's diverticulum? Laryngoscope, 2016; doi: 10.1002/lary.25975.

- Shahawy S, Janisiewicz A, Annino D, et al.: A comparative study of outcomes for endoscopic diverticulotomy versus external diverticulectomy. Otolaryngol Heas Neck Surg. 2014; 15(4):646-651. 
Surgical treatment of Zenker's diverticulum. Is the open approach really outdated?

- Sonbare DJ.: Pulsion Diverticulum of the Oesophagus : More than just an Out Pouch. Indian J Surg. 2015; 77(1):44-8. doi:10.1007/s12262-013$0955-8$

- Verdonck J, Morton RP.: Systematic review on treatment of Zenker's diverticulum.

- Eur Arch Otorhinolaryngol. 2015; 272(11):3095-107. doi:
- Visser L.J., Hardillo J.A.U., Monserez D.A., et al.: Zenker's diverticulum: Rotterdam experience. Eur Arch Otorhinolaryngol 2016; 273:2755-2763.

- Yuan Y, Zhao YF, Hu Y, et al.: Surgical treatment of Zenker's diverticulum. Dig Surg 2013; 30(3):207-18.

doi:10.1159/000351433.

10.1007/s004405-014-3247-4. 
Medical Research Archives, Vol. 4, Issue 8, December 2016

Surgical treatment of Zenker's diverticulum. Is the open approach really outdated?
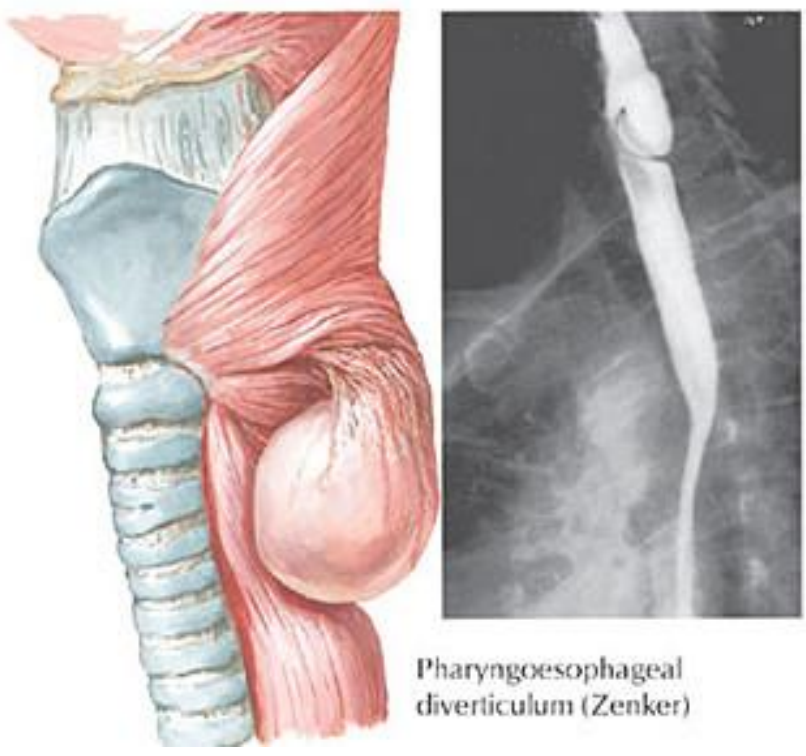

Pharyngoesophageal

diverticulum (Zenker)

Figure 1. Anatomy of Zenker's diverticulum. Source: nature.com

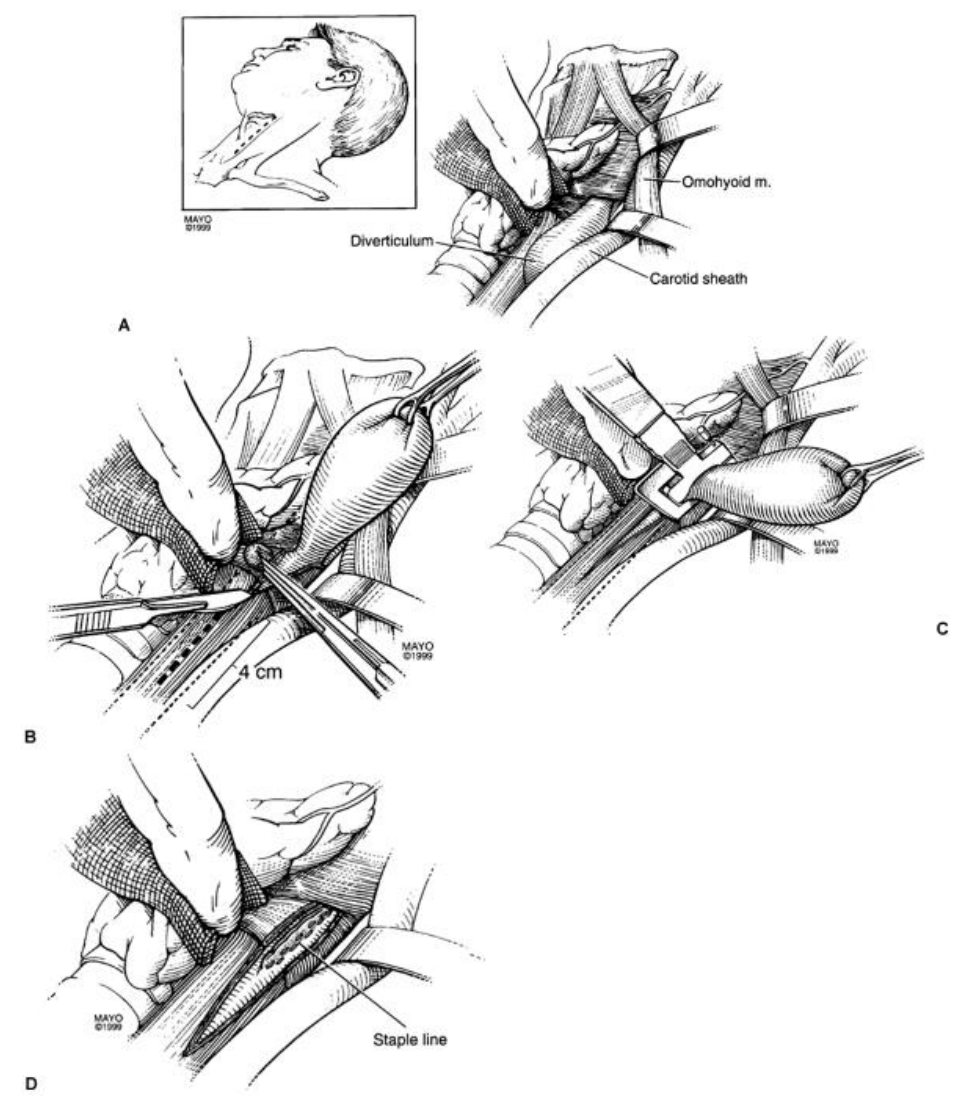

Figure 2. Transcervical resection of Zenker's diverticulum. Source: Shields Th.W 